\title{
Genome Sequencing Identifies Previously Unrecognized Klebsiella pneumoniae Outbreaks in Neonatal Intensive Care Units in the Philippines
}

Celia C. Carlos ${ }^{1}$, Melissa Ana L. Masim¹, Marietta L. Lagrada ${ }^{1}$, June M. Gayeta ${ }^{1}$, Polle Krystle V.

Macaranas $^{1}$, Sonia B. Sia ${ }^{1}$, Maria Adelina M. Facun ${ }^{1}$, Janziel Fiel C. Palarca ${ }^{1}$, Agnettah M. Olorosa ${ }^{1}$, Gicell Anne C. Cueno' ${ }^{1}$, Monica Abrudan², Khalil Abudahab², Silvia Argimón², Mihir Kekre², Anthony Underwood ${ }^{2}$, John Stelling ${ }^{3}$, David M. Aanensen ${ }^{2,4}$, and the NIHR Global Health Research Unit on Genomic Surveillance of Antimicrobial Resistance ${ }^{a}$

1 - Antimicrobial Resistance Surveillance Reference Laboratory, Research Institute for Tropical Medicine, Muntinlupa, the Philippines

2 - Centre for Genomic Pathogen Surveillance, Wellcome Genome Campus, Hinxton, Cambridge, United Kingdom

3 - Brigham and Women's Hospital, Boston, MA, USA

4 - Centre for Genomic Pathogen Surveillance, Big Data Institute, University of Oxford, Old Road Campus, Oxford, United Kingdom and Wellcome Genome Campus, Hinxton, UK

${ }^{a}$ Members of the NIHR Global Health Research Unit for the Genomic Surveillance of Antimicrobial Resistance are listed in the Acknowledgments.

Keywords: Whole genome sequencing, K. pneumoniae, outbreak detection, antimicrobial resistance

Running title: K. pneumoniae in the Philippines

Corresponding author: Celia C. Carlos, Antimicrobial Resistance Surveillance Reference Laboratory, Research Institute for Tropical Medicine, Muntinlupa, the Philippines. ccarlosphl@gmail.com. 
Alternate corresponding author: David M. Aanensen, Centre for Genomic Pathogen Surveillance, Wellcome Genome Campus, Hinxton, Cambridge, United Kingdom; Centre for Genomic Pathogen Surveillance, Li Ka Shing Centre for Health Information and Discovery, University of Oxford, Old Road Campus, Oxford, United Kingdom. david.aanensen@bdi.ox.ac.uk.

40-word summary: Whole genome sequencing identified three distinct previously unrecognized local outbreaks in a retrospective study in the Philippines, along with an epidemic plasmid carrying antimicrobial resistance genes, highlighting its importance in antimicrobial resistance surveillance, outbreak detection and infection control. 


\begin{abstract}
Background: Klebsiella pneumoniae is a critically important pathogen in the Philippines. Isolates are commonly resistant to at least two classes of antibiotics, yet mechanisms and spread of its resistance are not well studied.
\end{abstract}

Methods: A retrospective sequencing survey was performed on carbapenem-, extended spectrum beta-lactam- and cephalosporin-resistant Klebsiella pneumoniae isolated at 20 antimicrobial resistance (AMR) surveillance sentinel sites from 2015-2017. We characterized 259 isolates using biochemical methods, antimicrobial susceptibility testing, and whole genome sequencing (WGS). Known AMR mechanisms were identified. Potential outbreaks were investigated by detecting clusters from epidemiologic, phenotypic and genome-derived data.

Results: Prevalent AMR mechanisms detected include $b l a_{\mathrm{CTX}-\mathrm{M}-15}(76.8 \%)$ and bla $_{\mathrm{NDM}-1}(37.5 \%)$. An epidemic IncFII(Yp) plasmid carrying $b l a_{\mathrm{NDM}-1}$ was also detected in 46 isolates from 6 sentinel sites and 14 different sequence types (ST). This plasmid was also identified as the main vehicle of carbapenem resistance in 2 previously unrecognized local outbreaks of ST348 and ST283 at 2 different sentinel sites. A third local outbreak of ST397 was also identified but without the IncFII(Yp) plasmid. Isolates in each outbreak site showed identical STs, K- and O-loci, and similar resistance profiles and AMR genes. All outbreak isolates were collected from blood of children aged $<1$. Conclusion: WGS provided an in-depth understanding of the epidemiology of AMR in the Philippines, which was not possible with only phenotypic and epidemiologic data. The identification of three previously unrecognized Klebsiella outbreaks highlights the utility of WGS in outbreak detection, as well as its importance in public health and in implementing infection control programs. 


\section{FUNDING}

This work was supported by Official Development Assistance (ODA) funding from the National Institute of Health Research [Wellcome Trust grant number 206194].

This research was commissioned by the National Institute of Health Research using Official Development Assistance (ODA) funding. Views expressed in this publication are those of the authors and not necessarily those of the NHS, the National Institute for Health Research or the Department of Health.

\section{Conflict of Interest}

The authors: No reported conflicts of interest. All authors have submitted the ICMJE Form for Disclosure of Potential Conflicts of Interest. 


\section{Main manuscript}

\section{INTRODUCTION}

Antimicrobial resistance (AMR) is a serious threat to public health, as antimicrobial-resistant pathogens limit therapeutic options and result in increased morbidity and mortality [1]. AMR is also perceived as a threat to the achievement of the Sustainable Development Goals [2].

AMR surveillance has conventionally been performed by monitoring distribution of antimicrobialresistant pathogens in the population through phenotypic methods such as antimicrobial susceptibility testing (AST), standard culture, and bacterial serotyping for identification and characterization $[3,4]$. WHONET is also widely used for surveillance data collection and analysis, while SaTScan integrated with WHONET allows early and broad detection of event clusters using retrospective or prospective algorithms and other flexible spatial and/or temporal scan parameters [5-7].

In the Philippines, priorities and methods for surveillance of AMR, hospital-acquired, and community-acquired infections are determined by the local health care facility's infection control committee in coordination with the microbiology laboratory [8]. Hospital bacterial isolates, antibiograms, and clustering of patient groups within the hospital network are monitored and reviewed in a set time frame, and semi-annual infection rates and antibiograms are reported to clinicians and administrators [8]. Suspected outbreaks or the occurrence of uncharacteristically large numbers of cases are reported to the local office of the National Epidemiology Center - Department of Health for appropriate action $[8,9]$.

Bacterial typing based on phenotypes, however, fails to distinguish isolates that have the same resistance profiles (RP) and isolates belonging to closely related Klebsiella species [3, 10, 11]. The development of molecular methods such as pulsed-field gel electrophoresis and multi-locus sequence typing (MLST) enabled molecular detection of relatedness of isolates, but these are labor-intensive, time-consuming, expensive, and often of a low resolution insufficient for outbreak analysis because they assay variation at small proportions of the genome [12]. The decreasing costs of WGS can address these limitations by providing high-resolution subtypes of AMR pathogens $[13,14]$ and identifying AMR genes and their location on bacterial chromosomes or on plasmids $[15,16]$. Through 
genome-wide analysis, a more granular picture of the status of AMR can potentially be determined by demonstrating mechanisms of AMR, transmission of AMR genes, and relatedness of strains [14-16]. K. pneumoniae is considered a microorganism of public health importance in the Philippines and is classified as critically important by the World Health Organization [17]. It is one of the leading causes of hospital-acquired infections, especially among the immunocompromised [18, 19]. Carbapenemresistant K. pneumoniae was first isolated in the Philippines between 1992 and 1994 and has been molecularly characterized in recent years $[15,20]$. A local outbreak of carbapenem-resistant ST340 and its possible transmission through an IncFII(Yp) plasmid with bla $a_{\mathrm{NDM}-1}, r m t C$, and sull was also identified through retrospective WGS survey, leading to the hospital's review of infection control protocols and implementation of more stringent programs [15].

In this study, a retrospective sequencing survey was undertaken on carbapenem-, cephalosporinresistant, and/or extended spectrum beta-lactamase (ESBL)-producing $K$. pneumoniae isolated from 2015-2017 to provide genomic context for local prospective surveillance. WGS analysis identified 3 potential local outbreaks among neonates, which were confirmed by corroborating epidemiological information, such as close isolation times and overlapping locations.

\section{METHODS}

\section{Bacterial Isolates}

From 2295 Klebsiella isolates collected from 2015-2017 and stored in the Antimicrobial Resistance Surveillance Resistance Reference Laboratory (ARSRL) biobank, 263 (11.5\%) exhibited resistance to one or more of the following: carbapenems, third- and fourth-generation cephalosporins, and/or ESBLs. These were selected using metadata stored in WHONET. The isolates were retrieved and resuscitated on Tryptic Soy Broth and incubated overnight at $35^{\circ} \mathrm{C}$ for re-identification and re-testing of key phenotypic resistance (Supplementary Methods). Invasive specimens were prioritized when both invasive and non-invasive specimens representing identical combinations of RP, sentinel site, and year of collection were available.

\section{DNA Extraction and Whole-Genome Sequencing}


Genomic DNA was isolated using nexttec ${ }^{\mathrm{TM}}$ 1-Step DNA Isolation Kit for Bacteria (nexttec Biotechnologie GmbH, 20N. 904) in accordance with manufacturer's instructions. DNA was quantified using Quantifluor ${ }^{\circledR}$ dsDNA System (Promega, E2670) and Quantus ${ }^{\text {TM }}$ Fluorometer (Promega, E6150), and then sent to the Wellcome Trust Sanger Institute for sequencing using Illumina ${ }^{\circledR}$ HiSeq2500 platform with 100- or 250-base paired-end reads. A total of 259 (98.5\%) isolates passed quality control and were included in the study. Raw sequence data generated were deposited in the European Nucleotide Archive under the project accession PRJEB29738. Run accessions are provided in Microreact projects linked in the figure descriptions [21].

\section{Bioinformatics Analysis}

The following analyses were performed using pipelines developed within the National Institute for Health Research Global Health Research Unit on Genomic Surveillance of AMR: QC, De novo assembly, mapping based SNP phylogeny, AMR, and MLST predictions [22-29]. Briefly, the sequences were assembled using SPAdes and identified using BactInspector, and contamination was detected using confindr [30-32]. Fastqc, multiqc, and qualifyr were utilized for quality control [2729]. SNP-based phylogeny was generated by mapping reads to a reference sequence using BWA mem; variants were called and filtered using bcftools, and a maximum likelihood phylogeny was produced using IQTree [33-35].

Pathogenwatch was used to identify MLST, K-, and O-loci, virulence factors, and plasmid replicons [36]. AMR genes were predicted using ARIBA 2.14.4 in conjunction with the NCBI AMR acquired gene and PointFinder databases [37-38]. Sequences were analyzed using PlasmidFinder and mapped against $b l a_{\text {NDM }}$ plasmids p13ARS_MMH0112-3 and p14ARS_MMH0055-5 [39]. Only those with $\geq 95 \%$ coverage were considered as matched. Results were collated and uploaded to Microreact for visualization [21].

\section{Outbreak Analysis}

Isolates with identical locations, forming clusters in the phylogenetic tree, were inspected as potential outbreaks. Maximum-likelihood phylogenetic trees were generated for each cluster using reference genomes EuSCAPE_IL028, EuSCAPE_DK005, and SRR5514218. Epidemiologic data, AST results, and genotypic characteristics of isolates in each cluster were then investigated. Infection origin was 
computed based on date of admission and sample collection date. Positive isolates collected more than 2 days after hospital admission were determined to be hospital-acquired. Meanwhile, positive isolates collected 0-2 days before hospital admission were classified as community-acquired. WHONET-SaTSCan's space-time scan permutation simulated prospective was utilized to look for statistical clusters among isolates recovered from the same site in 2015-2017 and characterized by the same RPs as the outbreak isolates [7]. SaTScan analysis was also extended to 2019 to check the persistence of the RP at the sentinel site. A maximum cluster length of 365 days and a recurrence interval (RI) of >365 days were set to exclude random signals that occur by chance alone and are of limited epidemiologic significance [40].

\section{RESULTS}

\section{Isolate Distribution and Characteristics}

The 259 Klebsiella isolates were collected between 2015 and 2017 by 20 of 26 Antimicrobial Resistance Surveillance Program sentinel sites representing 16 of 17 regions (Supplementary Table 1). Isolates were submitted as carbapenem-resistant ( $\mathrm{n}=81,31.2 \%)$, carbapenem- and cephalosporinresistant ( $\mathrm{n}=58,22.4 \%)$, cephalosporin-resistant ( $\mathrm{n}=11,4.2 \%)$, ESBL-producing $(\mathrm{n}=62,23.9 \%)$, and ESBL-producing and cephalosporin-resistant ( $\mathrm{n}=47,18.1 \%)$ (Supplementary Table 2). Invasive isolates from blood $(\mathrm{n}=240,92.7 \%)$ and CSF $(\mathrm{n}=13,5.0 \%)$ were prioritized for WGS. A few non-invasive samples, i.e. urine $(n=3)$, sputum $(n=1)$, tracheal aspirate $(n=1)$, and umbilical cord $(n=1)$, were also analyzed based on their resistance profiles.

The majority of the isolates were from inpatients $(n=253,97.7 \%)$ and from hospital-acquired infections $(\mathrm{n}=171,66.0 \%)$. Isolates were collected from patients aged $<1$ to 93 years old, but most were from patients $<1$ year old $(n=145,56.0 \%)$, composed of $84.8 \%$ neonates $(0-28 d)$ and $15.2 \%$ infants (29d-11mo). Hence, infections were mostly detected in the neonatal department $(n=77$, 29.7\%). Other patients aged $<1$ were also admitted to the intensive care unit (ICU), pediatric, pediatric-ICU, mixed ward, and emergency departments.

\section{Species Identification and Sequence Type}


In silico species identification resulted in 214 (82.6\%) Klebsiella pneumoniae, 36 (13.9\%) Klebsiella quasipneumoniae subsp. similipneumoniae, and 9 (3.5\%) Klebsiella quasipneumoniae subsp.

quasipneumoniae (Figure 1). The 45 K. quasipneumoniae were also correlated with 45 identified bla $_{\mathrm{OKP}}$ genes, a $K$. quasipneumoniae chromosomal marker encoding ampicillin resistance [11]. The overlapping biochemical phenotypes and lack of a stable classifier among these closely related species may account for the inability of conventional laboratory techniques to definitively differentiate them $[11,41]$.

There were 102 different MLSTs predicted using ARIBA (Supplementary Figure 1). The most common were ST147 (n=23, 8.9\%), ST348 (n=19, 7.3\%), ST14 $(n=10,3.9 \%)$, and ST283 $(n=9$, 3.5\%) (Supplementary Table 3). Ten isolates were identified to have novel ST profiles.

\section{Capsular and Lipopolysaccharide Typing}

The K. pneumoniae capsule has been shown to be a key virulence determinant, suppressing host inflammatory response and providing resistance to antimicrobial peptides [42-44]. Capsular and lipopolysaccharide typing using the $\mathrm{K}$ and $\mathrm{O}$ loci identified 57 different $\mathrm{K}$ loci (KL) with good or higher confidence for 244 isolates, and $14 \mathrm{O}$ loci for 256 isolates (Supplementary Figure 2). The most prevalent KL types were KL62 (n=22, 8.5\%), KL10 ( $\mathrm{n}=20,7.7 \%)$, and KL64 ( $\mathrm{n}=16,6.2 \%)$. The most prevalent O loci were O1v1 (n=69, 26.6\%), O1v2 (n=34, 13.1\%), and O5 $(\mathrm{n}=31,12.0 \%)$.

\section{Resistance Profiles and AMR Genes}

A total of 93 RPs were observed, with the 4 most common accounting for $32.3 \%$ of the isolates (Supplementary Figure 1). RP-1, RP-3, and RP-4 were extremely drug resistant (XDR) RPs of $K$. pneumoniae and Escherichia coli reported to be expanding in the Philippines [15].

Known AMR mechanisms for carbapenem resistance, cephalosporin resistance, and ESBLs were identified (Supplementary Table 2). The most prevalent were the ESBL bla $a_{\mathrm{CTX-M}-15}$ gene ( $\mathrm{n}=199$, $76.8 \%)$ and the carbapenemase bla $_{\mathrm{NDM}-1}$ gene $(\mathrm{n}=97,37.5 \%)$. Other carbapenemase genes included $\operatorname{bla}_{\mathrm{NDM}-7}(\mathrm{n}=18,6.9 \%), \operatorname{bla}_{\mathrm{KPC}-2}(\mathrm{n}=4,1.5 \%)$, and bla $_{\mathrm{NDM}-9}(\mathrm{n}=1,0.4 \%)$. Nineteen isolates had unknown mechanisms but carried combinations of bla genes and ompK36 and ompK37 mutations, which have been linked to carbapenem resistance in Enterobacteriaceae [45]. 
$r m t C$, encoding high resistance to aminoglycosides, was also identified in 48 (18.9\%) isolates [46]. Other prevalent aminoglycoside resistance genes were: $a a c\left(6^{\prime}\right)-30 / a a c\left(6^{\prime}\right)-l b^{\prime}(81.5 \%), a a c\left(6^{\prime}\right)-I b$ (81.5\%), aac(3)-II (46.3\%), aph(3”)-Ib (47.9\%), and $\operatorname{aph}(6)-I d(47.9 \%)$.

Fluoroquinolone resistance genes $o q x A(95.4 \%)$ and $o q x B(95.4 \%)$ were also observed in most isolates, along with qnrBl (32.8\%), qnrS1 (33.2\%), qnrB6 (12.7\%), and qnrB4 (4.2\%). However, these genes only confer low level resistance or reduced susceptibility, which may not necessarily translate to phenotypic resistance [43]. Single gyrA mutations at codon $83(11.2 \%)$ and double mutations at codon 83 and 87 (9.3\%) were also found in some isolates. A single parC mutation cooccurred with 52 of 53 gyrA mutations (98.1\%). gyrA and parC mutations corresponded to ciprofloxacin resistance in 50 of 53 isolates (94.3\%). Sulfonamide and trimethoprim resistance genes dfrA (88.4\%), sull (70.7\%), and sul2 (58.3\%) were also detected.

\section{Inc Type Profiling}

A total of 32 different Inc types were observed among the 259 isolates (Supplementary Figure 1). $\operatorname{IncFIB}(\mathrm{K})(\mathrm{n}=201,21.4 \%)$ and $\operatorname{IncFII}(\mathrm{K})(\mathrm{n}=163,17.3 \%)$ were the most prevalent. Notable Inc types identified were IncFII(Yp) $(n=48,5.8 \%)$ and $\operatorname{IncX} 3(n=21,2.2 \%)$. A plasmid carrying IncFII(Yp), bla $a_{\mathrm{NDM}-1}, r m t C$, and sull (p13ARS_MMH0112-3) was previously linked to a nosocomial outbreak of K. pneumoniae ST340 in the Philippines [15]. The same IncFII(Yp) and AMR genes were also present in 46 of 48 IncFII(Yp)-positive isolates from 6 sentinel sites and 14 different STs (Supplementary Table 4). Short reads of all 46 isolates and one IncFII(Yp)-bla $a_{\mathrm{NDM}-7}$ mapped to the plasmid with $>95.0 \%$ coverage of the sequence length. There were $21 \mathrm{IncX} 3$ replicons found in the genomes of 19 out of 20 bla $_{\mathrm{NDM}-7}$-carrying isolates from 9 sentinel sites and 8 STs (Supplementary Table 4). Short reads of all 19 were mapped to the bla $a_{\mathrm{NDM}-7}$-carrying plasmid p14ARS_MMH0055-5 with 100\% coverage of the plasmid sequence [15]. These results suggest that both IncFII(Yp) and IncX3 plasmids have been widely circulating and conferring carbapenemase resistance to a diverse genetic background, including non-epidemic strains, in the Philippines [15]. This study is limited by short read data, hence further plasmid or long-read sequencing will be conducted to characterize other vehicles of AMR gene transmission.

\section{Local Outbreak of K. pneumoniae ST348}


WGS paired with epidemiological data provided a phylogenetic tree with 3 observed clusters (CMC, VSM, JLM) in 3 separate hospitals (Figure 1). This prompted an investigation to identify possible disease outbreaks. In all 3 clusters, all isolates were collected from blood and affected patients were all aged $<1$ year. Isolates in each cluster had identical MLST, capsular, and lipopolysaccharide types, and similar RPs and AMR genes (Figure 2). In addition, all the isolates in the CMC and JLM clusters carried plasmid p13ARS_MMH0112-3 (Supplementary Figure 3).

The largest cluster was observed in CMC, with 15 isolates collected between September 2016 and August 2017 identified as ST348 (Figure 2A). Most of the cases were hospital-acquired ( $\mathrm{n}=11$, $73.3 \%)$ with admissions to the neonatal ward (66.7\%), pediatric ward (20.0\%), and ICU (13.3\%). All isolates had the same KL62 and O1v1 loci and carried virulence factor yersiniabactin (ybt14). Mean pairwise SNP difference between these 15 isolates was 6.3 SNPs (range: 1-28), suggesting intrahospital transmission when compared with the 53.1 mean pairwise SNP differences (range: 1236) of other ST348 genomes from other sentinel sites that did not carry the bla $a_{\mathrm{NDM}}$ plasmid p13ARS_MMH0112-3 [47, 48].

There were 14 ST348 isolates (93.3\%) exhibiting RP-2, while 1 (6.7\%) was additionally resistant to CIP exhibiting RP-1, which was confirmed by retesting the isolate (Figure 2A). Since gyrA and parC mutations were absent, this might have been caused by unknown mechanisms or differences in gene expression despite having similar low-level fluoroquinolone resistance genes, such as oqx or aac(6')$I b-c r[49]$.

\section{Local Outbreak of K. pneumoniae ST397}

The second cluster was identified at sentinel site VSM, with 7 isolates collected from April 1 to May 2, 2016 identified as ST397. All patients were neonates, and cases were determined to be hospitalacquired (Figure 2B). KL158 and O1v1 loci and yersiniabactin gene $y b t 9$ were also identified in all isolates. The mean pairwise SNP difference between isolates was 3.9 SNPs (range: 1-8), indicating origin from a single transmission cluster [47, 48]. ST397 genomes from other hospitals were not available for comparison.

All isolates were ESBL-producing, with 57.1\% (n=4) exhibiting RP-5 (full profile is shown in Figure 2), corresponding to isolates carrying IncFIA(pBK30683). Loss of IncFIA(pBK30683) in 3 isolates 
was also concordant with observed loss of $a a c$, bla ${ }_{\mathrm{OXA}-1}, c a t, d f r A$, and $q n r B 1$, indicating that these genes may be carried on an IncFIA(pBK30683) plasmid.

\section{Local Outbreak of K. quasipneumoniae ST283}

The third cluster from JLM comprised 9 isolates collected from neonates in May 2016 to July 2017. Most cases were determined to be hospital-acquired (66.7\%) (Figure 2C). All isolates were previously identified as K. pneumoniae by biochemical methods, but as K. quasipneumoniae subsp. similipneumoniae ST283 by WGS. All had the same KL10 and O5 loci. The mean pairwise SNP difference between isolates was 3.75 SNPs (range: 0-10), suggesting intrahospital outbreak [47, 48]. ST283 genomes from other hospitals were not available for comparison.

Eight isolates (88.9\%) exhibited RP-1, while $1(11.1 \%)$ additionally showed intermediate susceptibility to CIP (RP-2), which was confirmed by retesting the isolates. This is possibly due to unknown mechanisms or differences in gene expression, despite having the same $a a c\left(6^{\prime}\right)-I b$-cr, $o q x A B$, and $q n r B 1$ genes conferring low level fluoroquinolone resistance, since $g y r A$ and parC mutations were not detected [49].

\section{WHONET-SaTSCan Analysis}

WHONET-SaTScan analysis of CMC Klebsiella isolates from 2015-2017 detected a statistically significant cluster for RP-2 (P<0.0013, RI=789d) (Table 1), which occurred in July 2017. This overlapped with the WGS-identified outbreak period in September 2016 to August 2017. Within this RP-2 cluster ( $n=10)$, we detected 3 of 4 ST37 isolates that mapped to the IncFII(Yp) plasmid (Supplementary Figure 3), and only 4 of 14 WGS-identified ST348 outbreak isolates (28.6\%). Extending the analysis to 2019 also showed an RP-1 cluster (n=4) occurred in July to August 2018, indicating possible persistence of outbreak strain or of the epidemic plasmid.

Clusters were also detected in VSM, but only in 2 of 4 RPs (RP-5, RP-6) (Table 1). RP-6 (n=7) and RP-5 (n=4) clusters occurring in March-April 2016 and April 2016 respectively also fell within the WGS-identified outbreak period from April 1 to May 2, 2016. Both clusters were statistically significant $(\mathrm{P}<0.001)$, but the RP-5 cluster showed strongest statistical significance $(\mathrm{P}<0.000096$, RI=10,369 days). Furthermore, only 5 of 7 WGS-identified ST397 outbreak isolates were detected in 
the WHONET-SaTScan clusters. Extending the analysis to 2019 also showed an RP-8 cluster ( $n=3$ ) occurring in December 2018, indicating the possible persistence of AMR in VSM.

Lastly, WHONET-SaTSCan analysis of all JLM Klebsiella isolates $(\mathrm{n}=1,562)$ generated no clusters for either outbreak RP, although RP-1 and RP-2 have been observed in 12 and 29 cases respectively from 2015-2019 (Supplementary Figure 4). This suggests an even distribution or a gradual increase of cases, rather than a sudden increase, which is indicative of an outbreak signal detected by SaTScan. Altogether, results showed that cluster analysis with WHONET-SaTScan and a fixed set of parameters may not detect all clusters, especially if they are comprised of few isolates exhibiting more than one RP. Scan type and spatial and/or temporal parameters may, however, be refined to detect clusters not only among RPs but also in specific wards.

\section{DISCUSSION}

We undertook a retrospective WGS survey of Klebsiella pneumoniae covering the years 2015-2017. We identified 3 outbreaks of Klebsiella among neonates in different hospitals in the Philippines, based on clusters observed in the phylogenetic tree, which resulted from combined epidemiologic and genotypic information. Average SNP differences in the 3 outbreaks were lower than the suggested thresholds of 16 and 21 SNPs for a K. pneumoniae intrahospital outbreak [47, 48].

Of the 3 outbreak strains, ST397 and ST283 were unique to VSM and JLM, respectively, but were nevertheless identified in other countries [50-53]. However, the same STs in other countries had differing AMR gene complements compared with the Philippine outbreak strains, which may therefore relate to local antimicrobial use practices [54].

For some isolates of the same outbreak strain, the complement of AMR genes differed among isolates of the same lineage, indicating distinct and potentially quite frequent gene-acquisition events, especially among hospital isolates. It is postulated that these AMR genes are acquired through selection due to antimicrobial exposure during hospital stay and may be carried by possibly epidemic plasmids circulating among non-epidemic strains, such as p13ARS_MMH0112-3, one of the main drivers of the JLM and CMC outbreaks [54]. Its occurrence in the genetic background of multiple STs within non-epidemic clones and at multiple sentinel sites suggests this is an epidemic plasmid that 
causes outbreaks among the immunocompromised, such as neonates in the ICU. However, further plasmid studies are needed to identify and characterize more AMR vehicles and modes of transmission.

There were no identified hypervirulent, extremely resistant $K$. pneumoniae, such as the epidemic KPC-producing ST258/ST11 clonal complex (CC258). However, possible XDR RPs (RP-1, RP-3, RP-4) $(n=63,24.2 \%)$ were among the most observed in this study, suggesting a possibly ongoing expansion [15]. As surveillance of AMR phenotypes is monitored, it may also be worthwhile to include surveillance of high-risk clones such as CC258 to predict invasive disease [54].

Using WGS, we were also able to distinguish what was phenotypically identified as K. pneumoniae to be K. quasipneumoniae. The phylogenetic tree of the 259 genomes in the Philippines showed deep branches separating the $K$. pneumoniae complex into $K$. pneumoniae and $K$. quasipneumoniae. $K$. quasipneumoniae is said to be less pathogenic than K. pneumoniae, which is more frequently associated with colonization or hospital-acquired infections [55].

The WGS pipeline was more efficient than WHONET-SaTScan at identifying potential outbreaks, since it was able to recognize the JLM outbreak, which SaTScan failed to do in a single run.

However, WHONET-SaTScan may still be a good complement for WGS as demonstrated in CMC, since it was able to tag non-outbreak strains carrying the same epidemic plasmid as the confirmed outbreak isolates. Clinical interpretation is still based on the insights of clinical and infection control staff [7]. Further, the method may be limited by hospital policies governing the choice of antibiotics for testing and reporting of results, which impacts on the configuration of WHONET [56]. On the other hand, sequence data can provide a broader range of genotype information about isolates, which allows better characterization through improved molecular resolution.

Review of resistance profiles in the 3 hospitals showed persistence of the possible XDR RPs in CMC and JLM, indicating that more aggressive infection control interventions may be necessary to control the continuing expansion. We have communicated with the infection control staff of both hospitals to alert them, and they have implemented aggressive measures to prevent future outbreaks. These outbreaks illustrate that the routine utility of both WHONET-SaTScan and WGS in context of 
retrospective data will enable real-time generation of alerts, early outbreak detection and investigation, and immediate infection control [57].

In conclusion, WGS provided a more in-depth understanding of AMR epidemiology in the Philippines. This resulted in the identification of 3 previously unrecognized local outbreaks of $K$. pneumoniae and $K$. quasipneumoniae among neonates in 3 distinct areas, which was not possible using phenotypic data alone. Sustaining WGS can improve public health services to identify patients with distinct AMR sequences who are at risk of treatment failure, to predict potential outbreaks, and to take action for their immediate control. 


\section{Acknowledgments}

Members of the NIHR Global Health Research Unit on Genomic Surveillance of Antimicrobial

Resistance: Harry Harste, Dawn Muddyman, Ben Taylor, Nicole Wheeler, and Sophia David of the

Centre for Genomic Pathogen Surveillance, Big Data Institute, University of Oxford, Old Road Campus, Oxford, United Kingdom and Wellcome Genome Campus, Hinxton, UK; Pilar DonadoGodoy, Johan Fabian Bernal, Alejandra Arevalo, Maria Fernanda Valencia, and Erik C. D. Osma Castro of the Colombian Integrated Program for Antimicrobial Resistance Surveillance - Coipars, CI Tibaitatá, Corporación Colombiana de Investigación Agropecuaria (AGROSAVIA), Tibaitatá Mosquera, Cundinamarca, Colombia; K. L. Ravikumar, Geetha Nagaraj, Varun Shamanna, Vandana Govindan, Akshata Prabhu, D. Sravani, M. R. Shincy, Steffimole Rose, and Ravishankar K.N of the Central Research Laboratory, Kempegowda Institute of Medical Sciences, Bengaluru, India; Iruka N Okeke, Anderson O. Oaikhena, Ayorinde O. Afolayan, Jolaade J Ajiboye, and Erkison Ewomazino Odih of the Department of Pharmaceutical Microbiology, Faculty of Pharmacy, University of Ibadan, Oyo State, Nigeria; Ali Molloy, alimolloy.com; and Carolin Vegvari, Imperial College London.

We are grateful to the members of the Antimicrobial Resistance Surveillance Program (Supplementary Note) that collected bacterial isolates and linked epidemiological data, especially to sentinel sites CMC, VSM, and JLM for cooperating and for providing additional information. We are also grateful to our project and support staff Elmer M. Herrera Jr., Laila T. Flores, Karis Lee D. Boehme, Michael F. Domingo, and our consultant Dr. Charmian M. Hufano for all their assistance. 


\section{REFERENCES}

1. O' Neil J. Review on Antibiotic resistance. Antimicrobial Resistance: Tackling a crisis for the health and wealth of nations. Review on Antimicrobial Resistance, 2014. Available at: https://amrreview.org/sites/default/files/AMR Review Paper - Tackling a crisis for the health and wealth of nations_1.pdf.

2. Jasovský D, Littmann J, Zorzet A, Cars O. Antimicrobial resistance-a threat to the world's sustainable development. Upsala Journal of Medical Sciences 2016; 121: 159-164.

3. Peacock SJ, Parkhill J, Brown NM. Changing the paradigm for hospital outbreak detection by leading with genomic surveillance of nosocomial pathogens. Microbiology (United Kingdom) 2018; 164: $1213-1219$.

4. World Health Organization. Global Antimicrobial Resistance Surveillance System (GLASS): molecular methods for antimicrobial resistance (AMR) diagnostics to enhance the Global Antimicrobial Resistance Surveillance System. World Health Organization, 2019.

5. World Health Organization. WHONET Software. Geneva: WHO, Available at: https://www.whonet.org/Docs/WHONET\%201.Getting\%20started.doc/. Accessed June 11, 2021. 6. SaTSCan - Software for the spatial, temporal, and space-time scan statistics. Available at: https://www.satscan.org/. Accessed June 10, 2021.

7. WHO Collaborating Centre for Surveillance of Antimicrobial Resistance. WHONET Cluster Detection with SaTSCan. 2006. Available at: https://whonet.org/Docs/WHONET 8.Cluster detection and SaTScan.doc. Accessed June 10, 2021.

8. National Center for Health Facility Development. National Standards in Infection Control for Healthcare Facilities. 2009. Available at:

https://www.doh.gov.ph/sites/default/files/publications/NATIONAL_STANDARDS_IN_INFECTIO N_CONTROL_FOR_HEALTH.pdf. Accessed June 10, 2021. 
9. World Health Organization. Disease Outbreaks. Available at:

https://www.who.int/teams/environment-climate-change-and-health/emergencies/disease-outbreaks/.

Accessed June 10, 2021.

10. Harris SR, Cartwright EJP, Török ME, et al. Whole-genome sequencing for analysis of an outbreak of methicillin-resistant Staphylococcus aureus: a descriptive study. The Lancet Infectious Diseases 2013; 13: 130-136.

11. Long SW, Linson SE, Saavedra MO, et al. Whole-genome sequencing of human clinical Klebsiella pneumoniae isolates reveals misidentification and misunderstandings of Klebsiella pneumoniae, Klebsiella variicola, and Klebsiella quasipneumoniae. Msphere 2017; 2.

12. Sabat AJ, Budimir A, Nashev D, et al. Overview of molecular typing methods for outbreak detection and epidemiological surveillance. Eurosurveillance 2013; 18: 20380.

13. Foxman B, Zhang L, Koopman JS, Manning SD, Marrs CF. Choosing an appropriate bacterial typing technique for epidemiologic studies. Epidemiologic Perspectives \& Innovations 2005; 2: 10.

14. Galarza PG, Alcalá B, Salcedo C, et al. Emergence of High Level Azithromycin-Resistant Neisseria gonorrhoeae Strain Isolated in Argentina. Sexually Transmitted Diseases 2009; 36: 787-788.

15. Argimón S, Masim MAL, Gayeta JM, et al. Integrating whole-genome sequencing within the National Antimicrobial Resistance Surveillance Program in the Philippines. Nature Communications $2020 ; 11$.

16. Tijet N, Faccone D, Rapoport M, et al. Molecular characteristics of mcr-1-carrying plasmids and new mcr-1 variant recovered from polyclonal clinical Escherichia coli from Argentina and Canada. PLoS One 2017; 12: e0180347.

17. World Health Organization. Global priority list of antibiotic-resistant bacteria to guide research, discovery, and development of new antibiotics. February 27, 2017. Im Internet: http://www who int/medicines/publications/WHO-PPL-Short_Summary_25Feb-ET_NM_WHO pdf 2018;

18. Jones RN. Microbial etiologies of hospital-acquired bacterial pneumonia and ventilator-associated bacterial pneumonia. Clinical Infectious Diseases 2010; 51: S81-S87.

19. Podschun R, Ullmann U. Klebsiella spp. as nosocomial pathogens: epidemiology, taxonomy, typing methods, and pathogenicity factors. Clinical Microbiology Reviews 1998; 11: 589-603. 
20. Chou A, Roa M, Evangelista MA, et al. Emergence of Klebsiella pneumoniae ST273 carrying bla NDM-7 and ST656 carrying bla NDM-1 in Manila, Philippines. Microbial Drug Resistance 2016; 22: $585-588$.

21. Argimón S, Abudahab K, Goater RJE, et al. Microreact: visualizing and sharing data for genomic epidemiology and phylogeography. Microbial Genomics 2016; 2.

22. Underwood A. MLST 1.0. Available at: https://gitlab.com/cgps/ghru/pipelines/dsl2/pipelines/mlst. Accessed June 10, 2021.

23. Underwood A. AMR Prediction 1.0. Available at: https://gitlab.com/cgps/ghru/pipelines/dsl2/pipelines/amr_prediction. Accessed June 10, 2021. 24. Underwood A. Mapping based SNP Phylogeny 1.2.2. Available at: https://gitlab.com/cgps/ghru/pipelines/snp_phylogeny. Accessed June 10, 2021.

25. Underwood A. De Novo Assembly 1.5.5. Available at: https://gitlab.com/cgps/ghru/pipelines/assembly. Accessed June 10, 2021.

26. Underwood A. GHRU (Genomic Surveillance of Antimicrobial Resistance) Retrospective 1 Bioinformatics Methods V.3. 2020. Available at: https://www.protocols.io/view/ghru-genomicsurveillance-of-antimicrobial-resista-bpbpmimn. Accessed June 10, 2021.

27. Underwood A. Qualifyr. Available at: https://gitlab.com/cgps/qualifyr. Accessed June 10, 2021. 28. Ewels P, Magnusson M, Lundin S, Käller M. MultiQC: summarize analysis results for multiple tools and samples in a single report. Bioinformatics 2016; 32: 3047-3048.

29. Babraham Bioinformatics. FastQC. Available at: https://www.bioinformatics.babraham.ac.uk/projects/fastqc/. Accessed June 10, 2021.

30. Bankevich A, Nurk S, Antipov D, et al. SPAdes: a new genome assembly algorithm and its applications to single-cell sequencing. Journal of Computational Biology 2012; 19: 455-477. 31. Underwood A. BactInspector. Available at: https://gitlab.com/antunderwood/bactinspector. Accessed June 10, 2021.

32. Low AJ, Koziol AG, Manninger PA, Blais B, Carrillo CD. ConFindr: rapid detection of intraspecies and cross-species contamination in bacterial whole-genome sequence data. PeerJ 2019; 7: e6995. 
33. Li H. Aligning sequence reads, clone sequences and assembly contigs with BWA-MEM. arXiv preprint arXiv:13033997 2013.

34. samtools. bcftools. Available at: https://github.com/samtools/bcftools. Accessed June 10, 2021. 35. Minh BQ, Schmidt HA, Chernomor O, et al. IQ-TREE 2: New models and efficient methods for phylogenetic inference in the genomic era. Molecular Biology and Evolution 2020; 37: 1530-1534. 36. The Centre of Genomic Pathogen Surveillance. Pathogenwatch | A Global Platform for Genomic Surveillance. 2018. Available at: https://pathogen.watch/. Accessed June 10, 2021.

37. Hunt M, Mather AE, Sánchez-Busó L, et al. ARIBA: rapid antimicrobial resistance genotyping directly from sequencing reads. Microbial Genomics 2017; 3.

38. Genomic Epidemiology. pointfinder_db. Available at:

https://bitbucket.org/genomicepidemiology/pointfinder_db/src/master/. Accessed June 10, 2021.

39. Carattoli A, Zankari E, Garciá-Fernández A, et al. In Silico detection and typing of plasmids using plasmidfinder and plasmid multilocus sequence typing. Antimicrobial Agents and Chemotherapy 2014; 58: 3895-3903.

40. Huang SS, Yokoe DS, Stelling J, et al. Automated detection of infectious disease outbreaks in hospitals: a retrospective cohort study. PLoS Med 2010; 7: e1000238.

41. Fontana L, Bonura E, Lyski Z, Messer W. The Brief Case: Klebsiella variicola-identifying the misidentified. Journal of Clinical Microbiology 2019; 57.

42. Wyres KL, Wick RR, Gorrie C, et al. Identification of Klebsiella capsule synthesis loci from whole genome data. Microbial Genomics 2016; 2. Available at: https://www.microbiologyresearch.org/content/journal/mgen/10.1099/mgen.0.000102.

43. Yoshida K, Matsumoto T, Tateda K, Uchida Kou, Tsujimoto S, Yamaguchi K. Role of bacterial capsule in local and systemic inflammatory responses of mice during pulmonary infection with Klebsiella pneumoniae. Journal of Medical Microbiology 2000; 49: 1003-1010.

44. Cortés G, Borrell N, de Astorza B, Gómez C, Sauleda J, Albertí S. Molecular analysis of the contribution of the capsular polysaccharide and the lipopolysaccharide $\mathrm{O}$ side chain to the virulence of Klebsiella pneumoniae in a murine model of pneumonia. Infection and Immunity 2002; 70: 25832590. 
45. Wozniak A, Villagra NA, Undabarrena A, et al. Porin alterations present in non-carbapenemaseproducing Enterobacteriaceae with high and intermediate levels of carbapenem resistance in Chile. Journal of Medical Microbiology 2012; 61: 1270-1279.

46. Wachino J, Yamane K, Shibayama K, et al. Novel Plasmid-Mediated 16S rRNA Methylase, RmtC, Found in a Proteus mirabilis Isolate Demonstrating Extraordinary High-Level Resistance against Various Aminoglycosides. Antimicrobial Agents and Chemotherapy 2006; 50: 178 LP - 184. Available at: http://aac.asm.org/content/50/1/178.abstract.

47. David S, Reuter S, Harris SR, et al. Epidemic of carbapenem-resistant Klebsiella pneumoniae in Europe is driven by nosocomial spread. Nature Microbiology 2019; 4: 1919-1929.

48. Ferrari C, Corbella M, Gaiarsa S, et al. Multiple Klebsiella pneumoniae KPC clones contribute to an extended hospital outbreak. Frontiers in Microbiology 2019; 10: 2767.

49. Jiang H-X, Song L, Liu J, et al. Multiple transmissible genes encoding fluoroquinolone and thirdgeneration cephalosporin resistance co-located in non-typhoidal Salmonella isolated from foodproducing animals in China. International Journal of Antimicrobial Agents 2014; 43: 242-247.

50. Yan JJ, Wang MC, Zheng PX, Tsai LH, Wu JJ. Associations of the major international high-risk resistant clones and virulent clones with specific ompK36 allele groups in Klebsiella pneumoniae in Taiwan. New Microbes and New Infections 2015; 5: 1-4.

51. Agyepong N, Govinden U, Owusu-Ofori A, et al. Genomic characterization of multidrug-resistant ESBL-producing Klebsiella pneumoniae isolated from a Ghanaian teaching hospital. International Journal of Infectious Diseases 2019; 85: 117-123.

52. Ocampo AM, Cienfuegos A v, Chen L, et al. High frequency of non-ST258 Klebsiella pneumoniae harboring KPC in tertiary-care hospitals from Medellín-Colombia. Available at: https://www.escmid.org/escmid_publications/escmid_elibrary/material/?mid=25419. Accessed June $10,2021$.

53. Aires CAM, Pereira PS, Rocha-de-Souza CM, Silveira MC, Carvalho-Assef APD, Asensi MD. Population Structure of KPC-2-Producing Klebsiella pneumoniae Isolated from Surveillance Rectal Swabs in Brazil. Microbial Drug Resistance 2020; 26: 652-660. 
54. Holt KE, Wertheim H, Zadoks RN, et al. Genomic analysis of diversity, population structure, virulence, and antimicrobial resistance in Klebsiella pneumoniae, an urgent threat to public health. Proceedings of the National Academy of Sciences 2015; 112: E3574-E3581.

55. Pons MJ, Marí-Almirall M, Ymaña B, et al. Spread of ST348 Klebsiella pneumoniae Producing NDM-1 in a Peruvian Hospital. Microorganisms 2020; 8: 1392.

56. Natale A, Stelling J, Meledandri M, Messenger LA, D'Ancona F. Use of WHONET-SaTScan system for simulated real-time detection of antimicrobial resistance clusters in a hospital in Italy, 2012 to 2014. Euro Surveill 2017; 22: 30484.

57. Latash J, Greene SK, Stavinsky F, et al. Salmonellosis Outbreak Detected by Automated Spatiotemporal Analysis-New York City, May-June 2019. Morbidity and Mortality Weekly Report 2020; 69: 815 . 
bioRxiv preprint doi: https://doi.org/10.1101/2021.06.22.449363; this version posted June 22,2021 . The copyright holder for this preprint

(which was not certified by peer review) is the author/funder, who has granted bioRxiv a license to display the preprint in perpetuity. It is made available under aCC-BY 4.0 International license.

\section{TABLES}

Table 1. Clusters detected by WHONET-SaTScan analysis of CMC and VSM Klebsiella isolates from 2015-2019.

No. of

$\mathbf{R P}$

No.

Resistance Profile

\section{Recurre}

nce

Interval
Cluster

p-value

Cluster Date

No. Outbreak

observed isolates in

cluster

\section{CMC}

$1 \quad$ AMP FOX CAZ CRO FEP IPM AMC $1,008 \quad 0.000992 \quad$ Jul-Aug $2018 \quad 4 \quad$ None

TZP GEN AMK CIP SXT

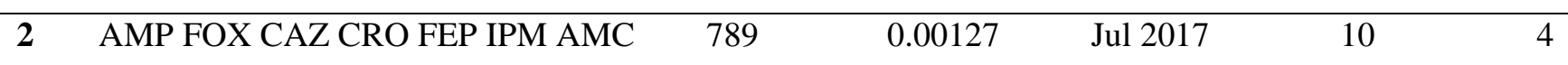

TZP GEN AMK SXT

\section{VSM}

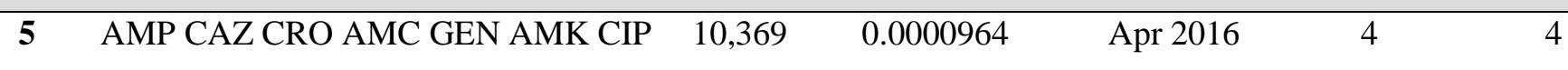

\begin{tabular}{|c|c|c|c|c|c|c|}
\hline 6 & AMP CAZ CRO AMC GEN CIP & 4,575 & 0.000219 & Mar-Apr 2016 & 7 & 1 \\
\hline 7 & AMP CAZ CRO & & - & - & - & - \\
\hline 8 & AMP CRO & 1,220 & 0.00082 & Dec 2018 & 3 & None \\
\hline
\end{tabular}




\section{FIGURE LEGENDS}

Figure 1. Phylogenetic tree of 259 Klebsiella isolates showing deep branches separating Kp I ( $K$. pneumoniae) and Kp II (K. quasipneumoniae). Clusters based on linked genotypic (ST, KL, and O locus types) data showed 3 clusters of possible NICU outbreaks in 3 separate hospitals. Most ST348 isolates were collected in CMC, while ST397 and ST283 were unique in VSM and JLM respectively. Maximum-likelihood tree was inferred from mapping genomes to reference K. pneumoniae strain K2044 (GCA_009497695.1). This interactive view is available at: https://microreact.org/project/p8oycZe8jyu3Aghc3EE99c.

Figure 2. Phylogenetic tree, linked epidemiological and genotypic data of outbreak isolates at 3 sentinel sites. A. Maximum-likelihood tree of CMC ST348 (n=15) isolates was inferred from mapping genomes to reference EuSCAPE_IL028. This interactive view is available at: https://microreact.org/project/p5amCjPTePU6ggNWanXSe1/cb996623. B. Maximum-likelihood tree of 7 VSM ST397 isolates was inferred from mapping genomes to reference EuSCAPE_DK005. This interactive view is available at: https://microreact.org/project/9K27BJqkWxtpsqhovaK3B3/98829bfe. C. Maximum-likelihood tree of 9 JLM ST283 isolates was inferred from mapping genomes to reference SRR5514218. This interactive view is available at: https://microreact.org/project/7MrkhkdfoWuCQjiUuRscuS/075c339d. Infection origin was described as either hospital-acquired infection (HAI) or community-acquired infection (CAI). Presence or absence of the following AMR genes was also described: bla $a_{\mathrm{NDM}}, r m t C$, bla $a_{\mathrm{CTX}-\mathrm{M}}, \operatorname{sull}$, aac(3)-Il, $a a c\left(6^{\prime}\right)-l b, b l a_{\mathrm{OXA}-1}, c a t, d f r A$, and $q n r B 1$. Presence of plasmid replicons IncFII(Yp) and IncFIA(pBK30683) was also described, along with plasmid match ( $\geq 95 \%$ coverage) to p13ARS_MMH0112-3. 
bioRxiv preprint doi: https://doi.org/10.1101/2021.06.22.449363; this version posted June 22, 2021. The copyright holder for this preprint (which was not certified by peer review) is the author/funder, who has granted bioRxiv a license to display the preprint in perpetuity. It is made available under aCC-BY 4.0 International license.

\section{FIGURES}

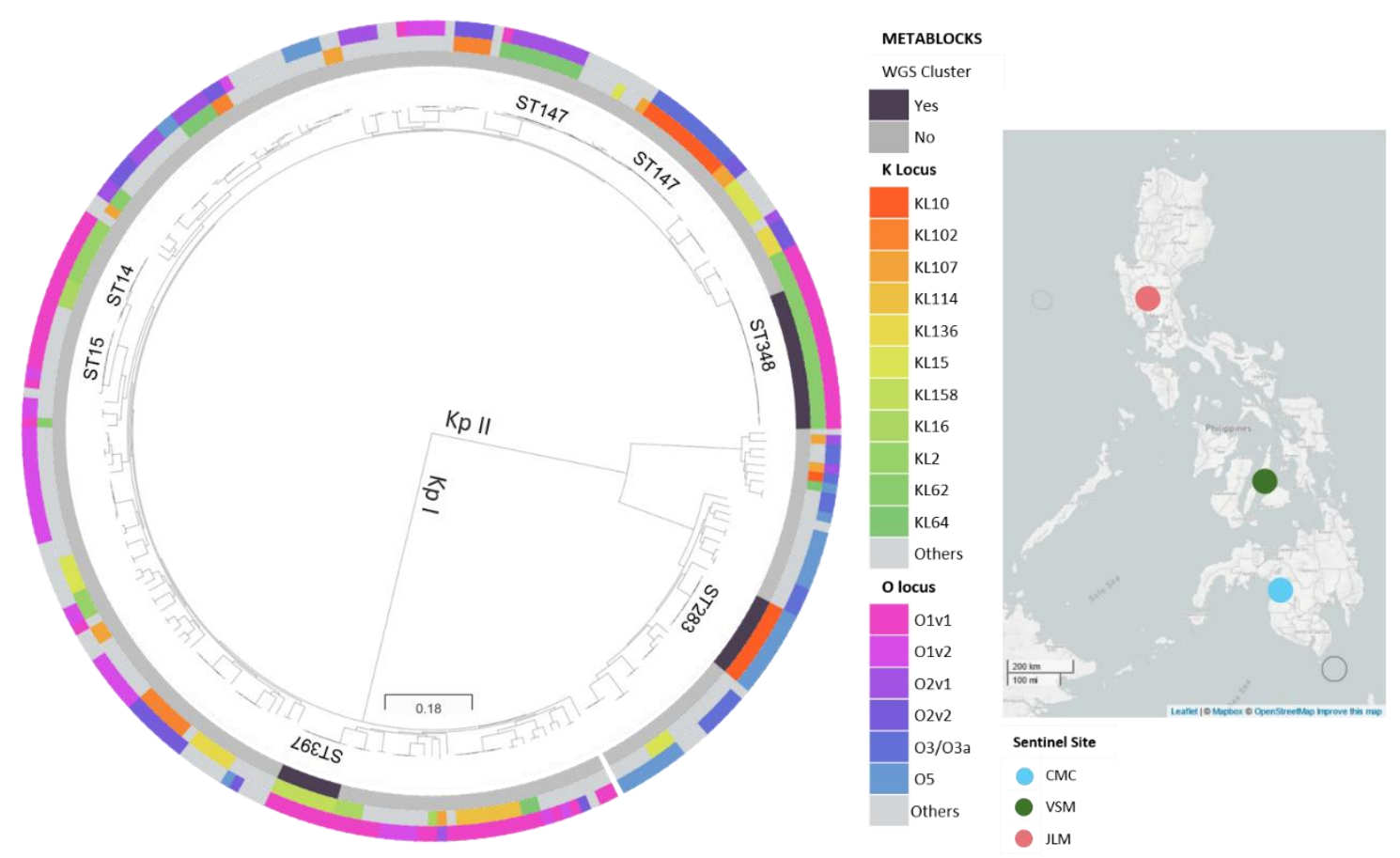

Figure 1. 
bioRxiv preprint doi: https://doi.org/10.1101/2021.06.22.449363; this version posted June 22, 2021. The copyright holder for this preprint (which was not certified by peer review) is the author/funder, who has granted bioRxiv a license to display the preprint in perpetuity. It is made available under aCC-BY 4.0 International license.

a)

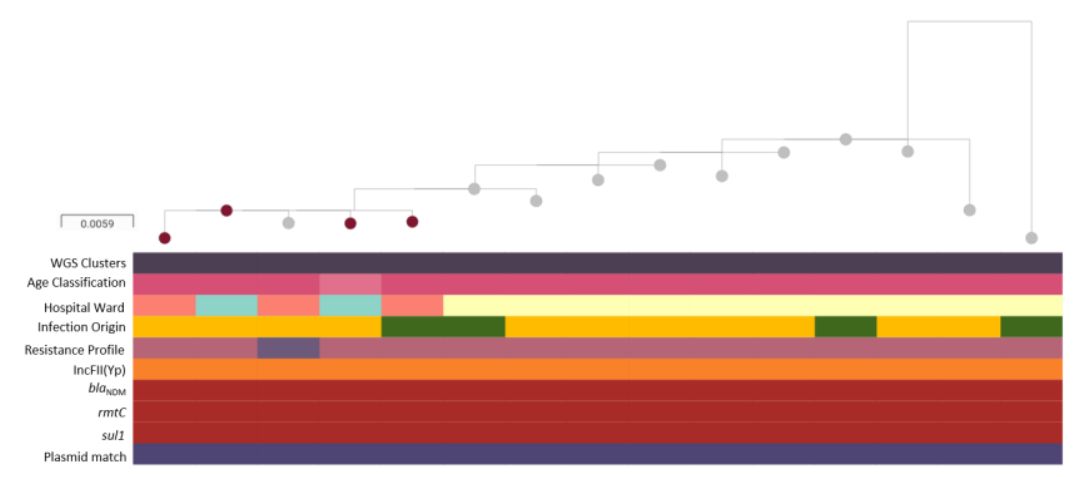

b)

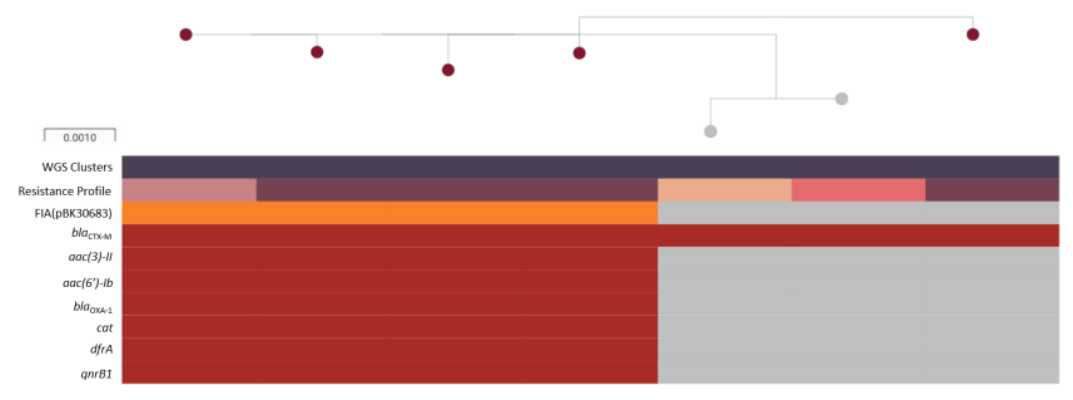

c)
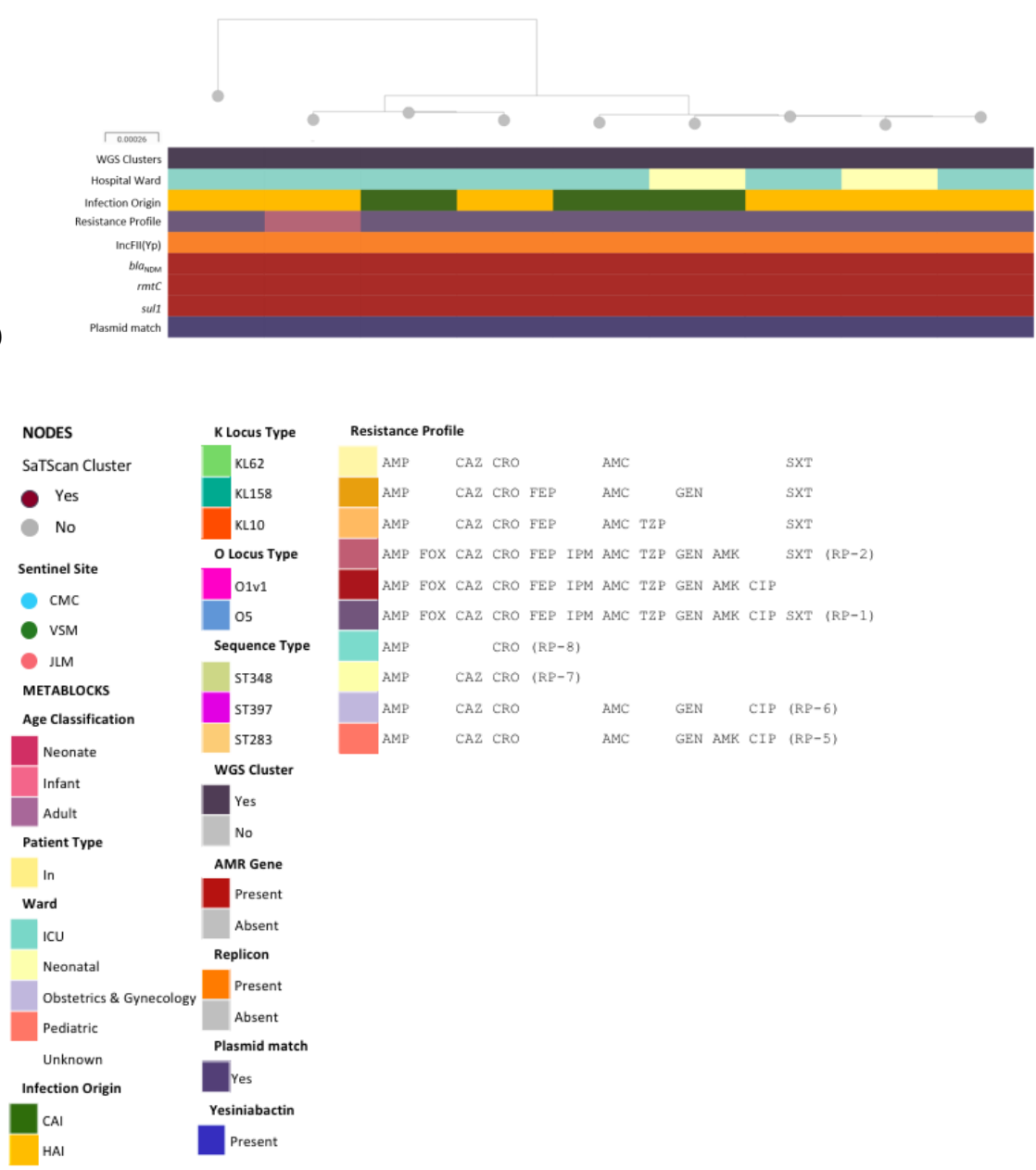

Figure 2. 